\title{
SEMINORMAL OPERATORS WITH COMPACT SELF-COMMUTATORS
}

\author{
KEVIN CLANCEY
}

ABstract. Putnam's inequality $\pi\left\|T^{*} T-T T^{*}\right\| \leqq \operatorname{meas}_{2}(\operatorname{sp}(T))$ is verified for seminormal operators $T$ when $T^{*} T-T T^{*}$ is compact. The spectrum of the seminormal singular integral operator defined for $f \in L^{2}(0,1)$ by

$$
T f(s)=s f(s)+i\left(\frac{\phi(s)}{\pi i} \int_{0}^{1} \frac{f(t) \bar{\phi}(t)}{s-t} d t\right), \quad s \in[0,1],
$$

where $\phi$ is any fixed essentially bounded measurable function on $[0,1]$, is computed.

1. An operator $A$ on a Hilbert space $\mathfrak{H}$ is called seminormal in case its selfadjoint self-commutator $D=A^{*} A-A A^{*}$ is positive semidefinite $(D \geqq 0)$ or negative semidefinite $(D \leqq 0)$. When $D \geqq 0$ the operator $A$ is said to be hyponormal. Putnam [7] has conjectured that if $A$ is seminormal then

$$
\pi\|D\| \leqq \operatorname{meas}_{2}(\operatorname{sp}(A))
$$

where meas $_{2}$ denotes Lebesgue planar measure and $\operatorname{sp}(A)$ denotes the spectrum of $A$. After this paper had been submitted for publication Putnam [6] discovered a proof of his remarkable inequality (1) for the general seminormal operator $A$. In $\$ 4$ we will establish (1) in the case where $D$ is compact. We will also determine the spectrum of the seminormal singular integral operator defined on $L^{2}(0,1)$ by

$$
T f(s)=s f(s)+i\left(\frac{\phi(s)}{\pi i} \int_{0}^{1} \frac{f(t) \phi(t)}{s-t} d t\right) \text { a.e. } s \in[0,1],
$$

where $f \in L^{2}(0,1)$ and $\phi$ is an $L^{\infty}(0,1)$ function. The singular integral in (2) is to be considered as a Cauchy principal value. The operator $T$ is hyponormal, indeed if $($,$) is the inner product in$ $L^{2}(0,1)$ then for $f \in L^{2}(0,1)$

$$
\left(T^{*} T-T T^{*}\right) f=\frac{2}{\pi}(f, \phi) \phi .
$$

We introduce for $0 \leqq x \leqq 1$ the notations

Received by the editors January 22, 1970.

AMS 1969 subject classifications. Primary 4615, 4710.

Key words and phrases. Seminormal operator, hyponormal operator, subnormal operator, singular integral. 


$$
\begin{aligned}
M_{x}^{ \pm} & =\operatorname{ess} \lim _{t \rightarrow x} \sup \left[ \pm|\phi(t)|^{2}\right] \\
& =\lim _{n \rightarrow \infty} \operatorname{ess} \sup \left[ \pm|\phi(t)|^{2}: t \in\left[x-\frac{1}{n}, x+\frac{1}{n}\right] \cap[0,1]\right] .
\end{aligned}
$$

In $\$ 3$ we will prove

THEOREM 1. If $T$ is the operator defined by (2) then

$$
\operatorname{sp}(T)=\left\{x+i y: 0 \leqq x \leqq 1, M_{x}^{-} \leqq y \leqq M_{x}^{+}\right\} .
$$

In [7] Putnam obtains Theorem 1 in the case where $\phi$ is Riemann integrable. Theorem 1 will yield (1) for all operators of the form (2). As is shown by an example in $\$ 3$ the inequality may be strict. The essential spectrum of operators of the form (2) when $\phi$ is smooth has been studied by Schwartz [9].

2. For an operator $B$ on $\mathcal{H}$ and complex number $z=x+i y$ we will write $B_{z}=B-z I$ and $B_{z}^{*}=B^{*}-\bar{z} I$. If $B=H+i J$ is the cartesian decomposition of $B$ where $H$ and $J$ are selfadjoint then $B_{z}=H_{x}+i J_{y}$. The fine structure of the spectrum of an operator $B$ will be needed; we will denote the compression spectrum of $B$ by $\Sigma(B)=\left\{z: B_{z}^{*} x=0\right.$, $x \in \mathcal{H}, x \neq 0\}$, the approximate point spectrum by $\pi(B)=\left\{z: B_{z} x_{n} \rightarrow 0\right.$, $\left.x_{n} \in \mathcal{F},\left\|x_{n}\right\|=1\right\}$, and the point spectrum of $B$ by $\pi_{0}(B)=\left\{z: B_{z} x=0\right.$, $x \in \mathcal{F}, x \neq 0\}$. Then clearly $\operatorname{sp}(B)=\Sigma(B) \cup \pi(B)$ and $\Sigma(B)=\{z: \bar{z}$ $\left.\in \pi_{0}\left(B^{*}\right)\right\}$. Moreover, it is well known that $\Sigma(B) \backslash \pi(B)$ is open (see, e.g. Halmos [3, p. 39]). In case $H$ is selfadjoint we will write for $\beta$ a Borel set $E(\beta)$ as the spectral projector of $H$ associated with $\beta$. If $A=H+i J$ is seminormal on $\mathfrak{F C}$ then $A_{\beta}=E(\beta) A E(\beta)$ is seminormal on $\mathfrak{H}_{\beta}=E(\beta) \mathcal{H C}$. It will be more convenient to write $A_{\beta}=\beta A \beta$ when there is only one spectral measure involved.

The following theorem is proved in Putnam [4, p. 46]:

TheOREM 2. Let $A=H+i J$ be seminormal then $\operatorname{pr}_{x}(\operatorname{sp}(A))=\operatorname{sp}(H)$ and $\mathrm{pr}_{y}(\mathrm{sp}(A))=\mathrm{sp}(J) .\left(\mathrm{pr}_{x}\right.$ and $\mathrm{pr}_{y}$ denote the projections on the $x$ and y axes respectively.)

We will also use the following lemma of Putnam [7]:

Lemma 1. Let $A=H+i J$ be hyponormal and $\Delta=[a, b]$, then $x$ $+i y \in \pi(A)$ for $x \in(a, b)$ if and only if $x+i y \in \pi\left(A_{\Delta}\right)$.

A selfadjoint operator $H$ with spectral measure $E(\beta)$ for $\beta$ a Borel set is said to be absolutely continuous in case the measure $\mu_{x}(\beta)$ $=\|E(\beta) x\|^{2}$ is absolutely continuous with respect to Lebesgue measure for each $x \in \mathfrak{H}$. 
We now prove the following lemma inspired by. Lemma 4 of Putnam [7].

LeMмA 2. Let $A=H+i J$ be hyponormal with compact self-commutator and $H$ absolutely continuous. If $\Delta=[a, b]$ and $z=x+i y \in$ $\Sigma\left(A_{\Delta}\right) \backslash \pi\left(A_{\Delta}\right)$ for $x \in(a, b)$ then $z \in \Sigma(A) \backslash \pi(A)$.

Proof. Set $c=\inf [s: z \in \Sigma([s, b] A[s, b]), s \leqq a]$. Clearly $c \leqq a$, if now $c$ is finite we will first show $z \in \Sigma([c, b] A[c, b])$ so that the inf is a min. Select $s_{n}$ decreasing to $c$ with $s_{n} \leqq a$ and $z \in \Sigma\left(\left[s_{n}, b\right] A\left[s_{n}, b\right]\right)$. Then there are $x_{n} \in \mathcal{H}_{\left[s_{n}, b\right]}$ with $\left\|x_{n}\right\|=1$ and

$$
\left[s_{n}, b\right] A_{z}^{*}\left[s_{n}, b\right] x_{n}=\left[s_{n}, b\right] A_{z}^{*} x_{n}=0 .
$$

Select from the $x_{n} \in \mathcal{F C}$ a weakly convergent subsequence $x_{n} \stackrel{\omega}{\rightarrow} q$. Now

$$
\left\|\left[s_{n}, b\right] A_{z}\left[s_{n}, b\right] x_{n}\right\|^{2}=\left(D x_{n}, x_{n}\right)
$$

so that if $q=0$ then $D x_{n} \rightarrow 0$ and then $\left[s_{n}, b\right] A_{z}\left[s_{n}, b\right] x_{n}$ would go to zero. We note that since (5) holds then $i\left[s_{n}, b\right] J_{y}\left[s_{n}, b\right] x_{n}=H_{x} x_{n}$ and so $\left[s_{n}, b\right] A_{z}\left[s_{n}, b\right] x_{n}=2 H_{x} x_{n}$ and therefore $H_{x} x_{n}$ also goes to zero. It follows that $\left[s_{n}, a\right] x_{n} \rightarrow 0$, indeed

$$
\begin{aligned}
\left\|H_{x} x_{n}\right\|^{2} & =\int_{\left[s_{n}, a\right]}(t-x)^{2} d\left\|E_{t} x_{n}\right\|^{2}+\int_{[a, b]}(t-x)^{2} d\left\|E_{t} x_{n}\right\|^{2} \\
& \geqq|a-x|^{2}\left\|\left[s_{n}, a\right] x_{n}\right\|^{2} .
\end{aligned}
$$

But then it follows that $[a, b] A_{2}[a, b] x_{n} \rightarrow 0$ for

$$
\begin{aligned}
\left\|\left[s_{n}, b\right] A_{z}\left[s_{n}, b\right] x_{n}\right\| & \geqq\left\|[a, b] A_{z}\left[s_{n}, b\right] x_{n}\right\| \\
& \geqq\left\|[a, b] A_{z}[a, b] x_{n}\right\|-\left\|[a, b] A_{z}\left[s_{n}, a\right] x_{n}\right\|
\end{aligned}
$$

where $\left[s_{n}, b\right] A_{z}\left[s_{n}, b\right] x_{n} \rightarrow 0$ and $\left[s_{n}, a\right] x_{n} \rightarrow 0$. Since lim sup $\left\|[a, b] x_{n}\right\|$ $>0$ and $z \notin \pi\left(A_{\Delta}\right)$ then $q$ cannot be zero. Clearly $q \in \mathcal{H}_{[c, b]}$ and since the operator $H$ is absolutely continuous then $\left[s_{n}, b\right] A z\left[s_{n}, b\right] p$ $\rightarrow[c, b] A_{z}[c, b] p$ for $p \in \mathcal{H}$. If now $p \in \mathcal{H}_{[c, b]}$ then

(9) $0=\left(\left[s_{n}, b\right] A_{z}^{*}\left[s_{n}, b\right] x_{n}, p\right)=\left(x_{n},\left[s_{n}, b\right] A_{z}\left[s_{n}, b\right] p\right) \rightarrow\left(q,[c, b] A_{z}[c, b] p\right)$.

Consequently $[c, b] A_{z}^{*}[c, b] q=0$ so $z \in \Sigma([c, b] A[c, b])$. On the other hand if $c$ is finite we may choose $s_{n}$ increasing to $c$ with $z \notin$ $\operatorname{sp}\left(\left[s_{n}, b\right] A\left[s_{n}, b\right]\right)$, certainly for $s_{n}<c$ we have $z \notin \Sigma\left(\left[s_{n}, b\right] A\left[s_{n}, b\right]\right)$ and by Lemma $1 z \notin \pi\left(\left[s_{n}, b\right] A\left[s_{n}, b\right]\right)$. Choose $\alpha>0$ so that the circle centered at $z$ of radius $\alpha$ is contained in the complement of $\operatorname{sp}(A)$ (if no such $\alpha$ exists then $z \in \Sigma(A) \backslash \pi(A)$ and we are done) and in the strip $(a, b) X(-\infty,+\infty)$. Since $\Sigma(B) \backslash \pi(B)$ is open for any operator $B$ and $s_{n}<c$ then the interior of this circle is contained in the comple- 
ment of $\operatorname{sp}\left(\left[s_{n}, b\right] A\left[s_{n}, b\right]\right)$ for all $n$. Now for $p \in \mathcal{H C}_{\left[\delta_{n}, b\right]}$ there results

$$
\left\|\left[s_{n}, b\right] A_{z}^{*}\left[s_{n}, b\right] p\right\|=\left\|\left[s_{n}, b\right] A_{z}^{*} p\right\| \geqq \alpha\|p\| .
$$

This follows since the resolvent $T_{z}^{-1}$ of a hyponormal operator $T$ is bounded in norm by $\operatorname{dist}^{-1}(z, \operatorname{sp}(T))$ where dist denotes distance. See, e.g. Stampfli [11]. Then for $p \in \mathcal{H}_{[c, b]}$ we have

$$
\left\|\left[s_{n}, b\right] A_{z}^{*} p\right\|>\alpha\|p\|
$$

and since $\left[s_{n}, b\right] A_{z}^{*} p \rightarrow[c, b] A_{z}^{*} p$ it follows that $z \notin \Sigma([c, b] A[c, b])$. The conclusion is that $c=-\infty$, but then if $m=\min \operatorname{sp}(H)$ we have $z \in \Sigma([m, b] A[m, b])$. Similarily set

$$
c^{\prime}=\sup [s: z \in \Sigma([m, s] A[m, s]), s<m]
$$

as above one concludes that $c^{\prime}=+\infty$. Then if $M=\max \operatorname{sp}(H)$ it follows that $z \in \Sigma([m, M] A[m, M])=\Sigma(A)$ and this completes the proof.

A hyponormal operator $A$ on $\mathcal{H}$ is said to have the normal reducing subspace $\mathfrak{T}$ in case $\mathfrak{T}$ reduces $A$ and the restriction of $A$ to $\mathfrak{T}$ is a normal operator. The following result is due to Stampfli [10] (see, also Clancey [1], Putnam [5], Yoshino [12]).

Theorem 3. Let $A$ be hyponormal and $z_{0} \in \operatorname{sp}(A)$. Assume that in some neighborhood $N$ of $z_{0}, \operatorname{sp}(A) \cap N$ is contained in a Jordan arc. Then $A=A_{1} \oplus A_{2}$ with $A_{1}$ normal and $\operatorname{sp}\left(A_{1}\right)=\operatorname{closure}[\operatorname{sp}(A) \cap N]$.

Lemma 3. Let $A=H+i J$ with $H$ absolutely continuous. Then for no $z_{0} \in \operatorname{sp}(A)$ does there exist a neighborhood $N$ of $z_{0}$ with $\operatorname{sp}(A) \cap N$ contained in a vertical segment.

PROOF. If such a point $z_{0}$ exists then by Theorem 3 there is a subspace $\mathscr{T}$ of $\mathcal{H}$ reducing $A$ on which $A$ acts like a normal operator, and the spectrum of the restriction $A_{1}$ of $A$ to $\mathfrak{T}$ is contained in a vertical segment. Then the real part $H_{1}$ of $A_{1}$ would have an eigenvalue and so would $H$ contradicting the fact that $H$ is absolutely continuous.

3. We turn now to a proof of Theorem 1.

Let $T$ be given as in (2) with $\phi \in L^{\infty}(0,1)$. The operator $T$ has the cartesian decomposition $H+i J$ where

$$
\begin{aligned}
H f(s)=s f(s) \text { and } \quad J f(s)=\frac{\phi(s)}{\pi i} \int_{0}^{1} \frac{f(t) \phi(t)}{s-t} d t & \\
& s \in[0,1], f \in L^{2}(0,1) .
\end{aligned}
$$


If now $\beta$ is any Borel set then Rosenblum [8] (see also Putnam $\left[4\right.$, p. 135]) has shown that $\operatorname{sp}\left(J_{\beta}\right)=\left[M_{\beta}^{-}, M_{\beta}^{+}\right]$where $M_{\beta}^{ \pm}=$ \pm ess sup $\left[|\phi(t)|^{2}: t \in \beta\right]$.

In case $z=x+i y \in\left\{s+i t: 0<s<1, M_{s}^{-}<t<M_{s}^{+}\right\}$it must be in $\operatorname{sp}(T)$. Otherwise for some $\delta>0$ the square $[x-\delta, x+\delta] X[y-\delta, y+\delta]$ is a subset of the complement of $\operatorname{sp}(T)$. In this case consider $T^{\prime}$ $=[x-\delta / 2, x+\delta / 2] T[x-\delta / 2, x+\delta / 2]$ then at least a part of one of the segments $\{x \pm \delta / 2\} X[y-\delta / 2, y+\delta / 2]$ is contained in $\operatorname{sp}\left(T^{\prime}\right)$ or else $\operatorname{pr}_{y}\left(\operatorname{sp}\left(T^{\prime}\right)\right)$ does not cover $\left[M_{\Delta}^{-}, M_{\Delta}^{+}\right]$where $\Delta=[x-\delta / 2, x+\delta / 2]$ contradicting Rosenblum's result. Since the real part of $T^{\prime}$ is absolutely continuous this is impossible (by Lemma 3 ) unless some portion of $(x-\delta / 2, x+\delta / 2) X(y-\delta / 2, y+\delta / 2)$ is in $\operatorname{sp}\left(T^{\prime}\right)$. From Lemma 1 we conclude this portion of $\operatorname{sp}\left(T^{\prime}\right)$ must be in $\Sigma\left(T^{\prime}\right) \backslash \pi\left(T^{\prime}\right)$ which is open. Consequently $z \in \Sigma\left(T^{\prime}\right)$ and since the commutator of $T$ is compact it follows from Lemma 2 that $z \in \Sigma(T)$. Putnam [7] has shown that $\operatorname{sp}(T)$ is a subset of $\left\{s+i t: 0 \leqq s \leqq 1, M_{s}^{-} \leqq t \leqq M_{s}^{+}\right\}$and therefore the proof of Theorem 1 is complete.

Note that we do not determine the fine structure of $\operatorname{sp}(T)$.

As was remarked above this determination of $\operatorname{sp}(T)$ yields the inequality (1) for all operators of the form (2) but as the following example shows the inequality may be strict. Consider a set $G$ in $[0,1]$ of measure $1 / 2$ with the property that $G$ intersects every proper open subinterval of $(0,1)$ and its complement in a set of positive measure. Put $\phi=$ characteristic function of $G$, then $M_{s}^{ \pm}= \pm 1$ for $s \in[0,1]$. Since

$$
\|D\|=(2 / \pi) \int_{0}^{1}|\phi(t)|^{2} d t=1 / \pi,
$$

then the inequality (1) is strict for this $T$.

4. We will begin with a technical result on compact sets in the plane. Let $K$ be a compact subset of the plane, we assume that $K$ is in the interior of some square $I_{0} X I_{0}$ where $I_{0}=[a, b]$ is a bounded interval. Define for any real number $x, I_{x}=\{y: x+i y \in K\}$ and $m(x)=\operatorname{meas}_{1}\left(I_{x}\right)$ where meas $_{1}$ denotes one-dimensional Lebesgue measure. For any interval $\Delta$ define $\Gamma(\Delta)=\Delta X(-\infty,+\infty)$ and $m(\Delta)=$ $\operatorname{meas}_{1}\left(\operatorname{pr}_{y}[\Gamma(\Delta) \cap K]\right)$.

LEMMA 4. Let $\Delta_{n}$ be a sequence of intervals with $\Delta_{n} \supseteq \Delta_{n+1}$, length of $\Delta_{n} \rightarrow 0$ and $x \in \Delta_{n}$ for all $n$, then $m\left(\Delta_{n}\right)$ decreases to $m(x)$.

Proof. Let $y \in I_{0} \backslash I_{x}, y \neq a, y \neq b$, then for some $\delta>0$ the square $[x-\delta, z+\delta] X[y-\delta, y+\delta]$ is contained in $I_{0} X I_{0} \backslash K$. For $n$ big enough 
so that $\Delta_{n} \subset(x-\delta, x+\delta)$ we have $y \notin \operatorname{pr}_{y}\left(\Gamma\left(\Delta_{n}\right) \cap K\right)$. Define $G_{n}$ $=\left\{y \in I_{0}: y \notin \mathrm{pr}_{y}\left(\Gamma\left(\Delta_{n}\right) \cap K\right)\right\}$. Clearly $G_{n}$ increases and we have just shown that $G_{n}$ increases to $I_{0} \backslash I_{x}$. So $m\left(\Delta_{n}\right)=\operatorname{meas}_{1}\left(I_{0}\right)-$ meas $_{1}\left(G_{n}\right)$ decreases to $\operatorname{meas}_{1}\left(I_{x}\right)=m(x)$. We will also use

Lemma 5. Let $A=H+i J$ be hyponormal with compact self-commutator and $H$ absolutely continuous. Let $\Delta=[a, b]$ be any closed interval, then $\operatorname{meas}_{1}\left(\operatorname{sp}\left(J_{\Delta}\right)\right)=\operatorname{meas}_{1}\left(\operatorname{pr}_{y}\left(\operatorname{sp}\left(A_{\Delta}\right)\right)\right) \leqq \operatorname{meas}_{1}\left[\operatorname{pr}_{y}(\Gamma(\Delta) \cap \operatorname{sp}(A))\right]$.

PRoof. The first equality follows from Theorem 2 . The inequality will certainly be established if we can show $\operatorname{sp}\left(A_{\Delta}\right) \subseteq \Gamma(\Delta) \cap \operatorname{sp}(A)$. Now by Lemmas 1 and 2 we have $\Gamma((a, b)) \cap \operatorname{sp}\left(A_{\Delta}\right) \subset \Gamma((a, b)) \cap \operatorname{sp}(A)$. Suppose now that $a+i y \in \operatorname{sp}\left(A_{\Delta}\right)$, then there must be a sequence $z_{n}=x_{n}+i y_{n} \rightarrow a+i y$ with $x_{n} \in(a, b)$ and $z_{n} \in \operatorname{sp}\left(A_{\Delta}\right)$ or else in some neighborhood of $a+i y, \operatorname{sp}\left(A_{\Delta}\right)$ would be contained in a vertical segment contradicting Lemma 3. But $z_{n} \in \operatorname{sp}(A)$ by Lemma 1 or Lemma 2 so that $a+i y \in \operatorname{sp}(A)$. A similar argument works in case $b+i y$ is in $\operatorname{sp}\left(A_{\Delta}\right)$ and this completes the proof. We can now prove

THEOREM 4. Let $T=H+i J$ be hyponormal with compact selfcommutator and with $H$ absolutely continuous. Then $\pi\left\|T^{*} T-T T^{*}\right\|$ $\leqq \operatorname{meas}_{2}(\mathrm{sp}(T))$.

Proof. We mimic Putnam's proof of Theorem 3.5.1 of [4, p. 49]. Let $\Delta=(a, b]$, then equation (3.5.7) in Putnam [4, p. 49] yields for $x \in \mathfrak{H}$

$$
(\pi)^{1 / 2}\left\|D^{1 / 2} E(\Delta) x\right\| \leqq\left[|\Delta| \operatorname{meas}_{1}\left(\operatorname{sp}\left(J_{\Delta}\right)\right)\right]^{1 / 2}\|E(\Delta) x\| .
$$

Define $Q(\Delta)=$ meas $_{1}\left(\operatorname{pr}_{y}(\Gamma(\Delta) \cap \operatorname{sp}(T))\right)$, then Lemma 5 yields $\operatorname{meas}_{1}\left(\operatorname{sp}\left(J_{\Delta}\right)\right) \leqq Q\left(\Delta^{\prime}\right)$ where $\Delta^{\prime}=[a, b]$. Therefore we have

$$
(\pi)^{1 / 2}\left\|D^{1 / 2} E(\Delta) x\right\| \leqq\left[|\Delta| Q\left(\Delta^{\prime}\right)\right]^{1 / 2}\|E(\Delta) x\| .
$$

If now $(c, d] \supset \operatorname{sp}(H)$ and $P: c=c_{0}<c_{1}<\ldots<c_{N}=d$ is a partition of $(c, d]$ into $\Delta_{k}=\left(c_{k-1}, c_{k}\right], k=1, \ldots, N$, then

$$
(\pi)^{1 / 2}\left\|D^{1 / 2} x\right\| \leqq\left\{\sum_{k=1}^{N}\left|\Delta_{k}\right| Q\left(\Delta_{k}^{\prime}\right)\right\}^{1 / 2}\|x\| .
$$

Now define $F(x)=Q\left(\Delta_{k}^{\prime}\right)$ on $\Delta_{k}$ so that

$$
\pi(D x, x) \leqq\left\{\int_{0}^{d} F(x) d x\right\}\|x\|^{2} .
$$

Select now a sequence of partitions $P_{n}$ with $P_{n+1}$ a refinement of $P_{n}$ and so that the norm of $P_{n}$ goes to zero. If $F_{n}(x)$ corresponds to $P_{n}$ as $F(x)$ did to $P$ then $F_{n}(x)$ decreases to $m(x)$ [for $\left.K=\mathrm{sp}(T)\right]$ a.e. (the 
a.e. takes care of the endpoints of $P_{n}$ ). By the Lebesgue Monotone Convergence Theorem it follows from (16) that

$$
\pi(D x, x) \leqq\left\{\int_{c}^{d} m(x) d x\right\}\|x\|^{2}=\operatorname{meas}_{2}(\operatorname{sp}(T))\|x\|^{2} .
$$

The following theorem due to Putnam [4, p. 42] shows that absolute continuity of $H$ can be attained.

TheOREM 5. Let $T=H+i J$ be seminormal on the Hilbert space $\mathcal{H}$. Let $\mathfrak{T}=\mathfrak{M}_{T}$ denote the smallest subspace of $\mathfrak{H C}$ containing the range of $D$ and invariant under both $T$ and $T^{*}$, then the restriction of $T$ to $\mathbb{N}$ has absolutely continuous real part, moreover, $T$ on the orthogonal complement of $\mathfrak{T l}$ is normal.

Then Theorems 4 and 5 combine to give

THEOREM 6. Let $T=H+i J$ be seminormal with compact selfcommutator, then $\pi\left\|T^{*} T-T T^{*}\right\| \leqq \operatorname{meas}_{2}(\operatorname{sp}(T))$.

Obviously it follows from Theorem 6 that if $T$ is seminormal with compact self-commutator and $\operatorname{meas}_{2}(\operatorname{sp}(T))=0$ then $T$ is normal.

There exists examples of seminormal operators with compact selfcommutators whose spectra have holes. Let $\left\{g_{i}\right\}_{i=-\infty}^{+\infty}$ be an orthonormal basis for $\mathcal{H C}$ and set $T g_{i}=a_{i} g_{i+1}, i=0, \pm 1, \pm 2, \cdots$, where

$$
\begin{aligned}
0 & <m=\lim _{i \rightarrow-\infty}\left|a_{i}\right| \leqq \cdots \leqq\left|a_{i}\right| \leqq\left|a_{i+1}\right| \\
& \leqq \cdots \leqq \lim _{i \rightarrow \infty}\left|a_{i}\right|=M<\infty .
\end{aligned}
$$

Then $T$ is hyponormal indeed if $D=T^{*} T-T T^{*}$ then $D g_{i}$ $=\left[\left|a_{i+1}\right|^{2}-\left|a_{i}\right|^{2}\right] g_{i}$ which is clearly compact. Also $\operatorname{sp}(T)=\{z: m$ $\leqq|z| \leqq M\}$ and for $z \notin \operatorname{sp}(T)$ the operator $T_{z}^{-1}$ is hyponormal with compact self-commutator. It follows that (1) holds for the operators $T_{z}^{-1}$. Compare with the result in Clancey [1].

\section{REFERENCES}

1. K. Clancey, Putnam's inequality for the annulus, Bull. Amer. Math. Soc. 76 (1970), 93-95.

2. - Spectral properties of semi-normal operators, Ph. D. Thesis, Purdue University, Lafayette, Ind., 1969.

3. P. R. Halmos, A Hilbert space problem book, Van Nostrand, Princeton, N. J., 1967. MR $34 \# 8178$.

4. C. R. Putnam, Commutation properties of Hilbert space operators and related topics, Ergebnisse der Mathematik und ihrer Grenzgebiete, Band 36, Springer-Verlag, New York, 1967. MR 36 \#707. 
5. - Hyponormal operators and low density spectra, Math. Z. 106 (1968), 167-169. MR $38 \# 564$.

6. - - An inequality for the area of hyponormal spectra, (to appear).

7. - The spectra of semi-normal singular integral operators, Canad. J. Math. (to appear).

8. M. Rosenblum, A spectral theory for self-adjoint singular integral operators, Amer. J. Math. 88 (1966), 314-328. MR 33 \#6453.

9. J. Schwartz, Some results on the spectra and spectral resolutions of a class of singular integral operators, Comm. Pure Appl. Math. 15 (1962), 75-90. MR 29 \#480.

10. J. G. Stampfli, A local spectral theory for operators, J. Functional Analysis 4 (1969), 1-10.

11. - Hyponormal operators and spectral density, Trans. Amer. Math. Soc. 117 (1965), 469-476. MR 30 \#3375.

12. T. Yoshino, Spectral resolution of a hyponormal operator with the spectrum on a curve, Tôhoku Math. J. (2) 19 (1967), 86-97. MR 35 \#7154.

Carleton University, Ottawa, Canada 